\title{
Philosophiques
}

\section{La connaissance historique via l'interliaison psychique : Wilhelm Dilthey}

\section{Laurent Giroux}

Volume 7, numéro 2, octobre 1980

URI : https://id.erudit.org/iderudit/203138ar

DOI : https://doi.org/10.7202/203138ar

Aller au sommaire du numéro

Éditeur(s)

Société de philosophie du Québec

ISSN

0316-2923 (imprimé)

1492-1391 (numérique)

Découvrir la revue

Citer cet article

Giroux, L. (1980). La connaissance historique via l'interliaison psychique :

Wilhelm Dilthey. Philosophiques, 7(2), 189-209. https://doi.org/10.7202/203138ar d'utilisation que vous pouvez consulter en ligne.

https://apropos.erudit.org/fr/usagers/politique-dutilisation/ 


\title{
LA CONNAISSANCE HISTORIQUE VIA L'INTERLIAISON PSYCHIQUE : WILHELM DILTHEY
}

\author{
par Laurent Giroux
}

Le courant de pensée surnommé « idéaliste ${ }^{1}$ - par opposition au positivisme - en épistémologie de la connaissance historique fut représenté en Allemagne par l'école historique qui a culminé, à la fin du siècle dernier dans l'œuvre de Wilhelm Dilthey. On le retrouve au début du $\mathrm{XX}^{\mathrm{e}}$ siècle en Italie chez Benedetto Croce et, quelques années plus tard, en milieu anglo-saxon avec Collingwood dont la théorie du "re-enactment " provoque encore la réflexion des épistémologues. Le Professeur William Dray lui a consacré récemment un article important dans la revue Dialogue (vol. XVII, $\mathrm{n}^{\circ} 4$, décembre 1978 , p. 659-682) et revient tout juste d'un congé sabbatique à Oxford où il travaillait à la rédaction d'un ouvrage sur le même auteur.

Ce qui de prime abord semble commun à tous ces "idéalistes", c'est la conviction que le sujet pensant a une connaissance immédiate de sa propre expérience en tant que sujet pensant et que cette pensée de soi donne accès au monde de l'esprit en général. Cette conviction, sous sa forme la plus élémentaire, remonte à tout le moins à Descartes et, bien que Kant ait démontré de façon assez convaincante que toute connaissance, même de soi, est médiatisée par les formes de la sensibilité et les structures de l'entendement, cette conviction persiste et hante la réflexion épistémologique. Ce n'est donc pas en un sens univoque que nos idéalistes seraient idéalistes, si toutefois ils le sont, ou peut-être seraient-ils idéalistes sans être des idéalistes trancendantaux.

1. "Idéaliste » semble être la dénomination préférée des positivistes pour désigner tout ce qui n'est pas positiviste. 
Quoi qu'il en soit, même si cette immédiateté de la connaissance de soi était admise sans discussion, la connaissance d'autres «soi " pose des problèmes quasi insurmontables, surtout si on veut à tout prix lui reconnaître une valeur scientifique en un sens tant soit peu acceptable. Car si l'expérience de l'autre doit devenir expérience immédiate de soi pour être connue, elle perd son altérité et ne se distingue plus de l'expérience propre de soi. Si, au contraire, elle demeure autre, on ne voit plus trop bien comment la connaissance de soi en tant que soi peut servir de tremplin pour aller la rejoindre en tant qu'autre. Dilthey posera le problème dans les termes suivants :

La possibilité de saisir un autre est tout d'abord l'un des problèmes les plus profonds de la théorie de la connaissance.

Comment une individualité peut-elle amener à une compréhension objective universellement valable une expression de vie individuelle étrangère qui lui est donnée par les sens ? (GW, p. 334).

Ceci nous amène à nous poser à propos de Dilthey un certain nombre de questions qu'il faudra éventuellement reprendre dans des travaux ultérieurs au sujet de Croce et de Collingwood.

Ces questions sont les suivantes :

$1^{\circ}$ Comment Dilthey justifie-t-il sa conviction que l'expérience interne est plus immédiate ou plus immédiatement connue que l'expérience externe?

$2^{\circ}$ Comment cette immédiateté, telle que conçue par Dilthey, peut-elle servir de fondement et de tremplin à une expérience d'autrui et, par cette voie, à la connaissance du passé historique?

$3^{\circ}$ À quel titre cette connaissance peut-elle être considérée comme science?

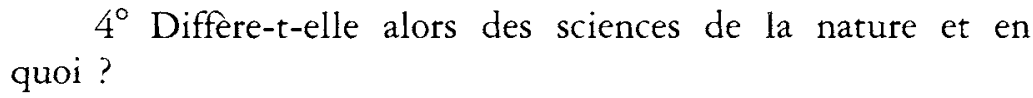

$4^{\circ}$ Differe-t-elle alors des sciences de la nature et en quoi ?

J'entrevois la réponse à ces questions dans la présence chez Dilthey d'un référent idéaliste (kantien et hégélien à la fois) 
transposé du plan transcendantal au niveau de la vie psychique (Seelenleben), c'est-à-dire sur un plan phénoménologique. Cette hypothèse de départ peut, du reste, s'appuyer sur une remarque explicite de Dilthey lui-même : "À l'encontre de la doctrine idéaliste de la raison, je ne me suis pas reporté vers un a priori de l'entendement théorique ou de la raison pratique qui serait fondé dans un Je pur, mais vers les relations structurelles contenues dans l'interliaison psychique et susceptibles d'être exposées. Cette interliaison structurelle forme le soubassement du processus de connaissance "(AGW 13, note 1 ; GW 151). Dilthey reconnaît en outre aux Investigations logiques de Husserl le mérite de lui avoir rendu possible ce « dé-part » de l'idéalisme.

Reprenons maintenant les différentes questions formulées ci-dessus et voyons comment Dilthey mène la discussion sur chacun des points qu'elles soulèvent. Il conviendra ensuite de terminer notre exposé par un ré-évaluation des critiques particulièrement significatives de Gadamer et de Habermas.

Des textes qui touchent à ces questions, il semble que nous puissions dégager les cinq propositions suivantes.

$1^{\circ}$ Les Erlebnisse ou expériences internes vécues sont des données immédiates et immédiatement perçues par la conscience. Le terme Erlebnis veut justement suggérer le caractère particulier des expériences intérieures dont la nature même consiste à être éprouvées ou vécues.

$2^{\circ}$ L'interliaison des Erlebnisse en ensembles structurels reliés (Strukturzusammenbang) est un phénomène spontané et immanent à ces expériences vécues elles-mêmes, ét non un cadre théorique qui leur serait imposé de l'extérieur.

$3^{\circ}$ Cette interliaison n'en est pas moins une construction ou plutôt une structuration opérée par le sujet conscient en accord avec certaines normes ou lois (Gesetzmässigkeit).

$4^{\circ}$ L'unité de l'interliaison psychique est d'ordre téléologique. C'est le but visé qui les coordonne de l'intérieur en une totalité signifiante.

$5^{\circ}$ C'est cette interliaison ou ensemble relié d'Erlebnisse qui rend possible la connaissance d'autrui en particulier et de l'histoire humaine en général. 
La formulation même des énoncés diltheyiens permet de saisir d'entrée de jeu avec quel type de problème nous sommes confrontés à l'intérieur de cette théorie. Si les sciences dites "de l'esprit " ou "morales" ont leur point de départ dans des Erlebnisse tels que définis, et que ces Erlebnisse s'organisent spontanément en ensembles reliés et unifiés suivant un axe téléologique, ces interliaisons étant elles-mêmes des Erlebnisse, est-ce l'immédiateté même du vécu qui permet d'entrer dans l'univers de l'esprit ou son organisation en totalités de signification ou bien encore les lois selon lesquelles cette organisation s'effectue ? "Comment le savoir de l'interliaison historique contenu dans la science de l'histoire qui existe factuellement est-elle possible?" $(\mathrm{AGW}, 109)^{2}$. Notre question porte donc sur la valeur de la réponse que Dilthey apporte à sa propre question. Ce qui nous oblige à examiner d'abord les textes où est définie la nature de l'interliaison interne des faits psychiques.

À la lecture des écrits de Dilthey, dans la mesure où l'on est préoccupé par les questions soulevées ici, deux points ressortent avec une certaine insistance :

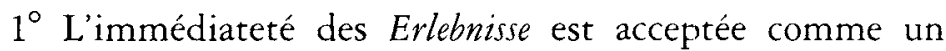
postulat de départ non remis en question et justifié seulement au passage par des raisons plutôt fragiles qui ne parviennent pas à écarter tous les doutes. Toutefois, cette faiblesse n'est pas nécessairement fatale pour la théorie, comme il apparaîtra plus loin.

$2^{\circ}$ L'interliaison psychique, bien qu'elle soit immanente aux Erlebnisse et elle-même un Erlebnis, est affectée d'un certain caractère transcendantal qui doit la rendre virtuellement apte à jouer un rôle dans la théorie de la connaissance. Il faut cependant s'assurer que son immanence ne prohibe pas au départ tout dépassement vers un type quelconque de généralisation ${ }^{3}$.

2. Cf. aussi les questions posées à la p. 254 .

3. Ce risque n'a pas échappé à Dilthey lui-même: "Nous nous trouvons donc en face du problème de savoit comment la structuration du monde de l'esprit dans le sujet rend possible un savoir de la réalité spirituelle. J'ai autrefois désigné cette tâche comme celle d'une critique de la raison historique " (AGW, 191). 
À propos du premier point, deux choses sont à noter :

Tout d'abord, il y a immédiateté pour moi de mes propres Erlebnisse parce qu'ici l'objet à connaître se confond avec la conscience connaissante elle-même :

La conscience d'un Erlebnis et sa constitution, son être-là-pourmoi et ce qui en lui est là pour moi sont un : l'Erlebnis ne se tient pas comme un objet vis-à-vis l'appréhendant, mais son être-là-pour-moi n'est pas distinct de ce qui en lui est là pour moi (AGW, 139).

Autrement dit son contenu (sa "constitution"), c'est son être-là-pour-moi lui-même. Il est difficile de voir en quoi cela privilégie l'Erlebnis par rapport à la perception externe qui est aussi un Erlebnis - un être-là-pour-moi - avec cette seule différence que son contenu y est posé comme extérieur au sujet percevant. Y a-t-il une différence de nature entre éprouver de la peine et voir le mercure rouge d'un thermomètre? La colonne rouge est aussi intérieure à ma perception que ma peine au sentiment que j'en ai.

Mais il y a plus. Bien que la connaissance d'Erlebnisse étrangers soit justifiée par ailleurs en raison de la similitude des esprits, comme nous le verrons ci-après, l'immédiateté de l'Erlebnis individuel semble ouvrir un accès à tout Erlebnis en général. Tout se passe comme s'il n'y avait pas seulement similitude des esprits, mais identité de l'Esprit, si bien qu'une fois l'entrée pratiquée par une porte, la sphère entière du monde spirituel se déploierait comme dans un miroir sous le regard. La raison qui en est donnée, c'est que ce type d'expérience n'a pas à passer par les canaux diversifiés des sens (GW, 264), raison étonnante sans doute, mais que Dilthey explique mieux dans un autre contexte où il montre, à l'aide d'un référent kantien, qu'il n'y a pas à proprement parler d'objets psychiques, puisque le concept même d'objet implique la synthèse des impressions des sens en un tout distinct du soi et indépendant de lui dans la continuité spatiale: "Seules les impressions des sens peuvent alors se relier en objet " (GW, 248). Il en va autrement des données psychiques :

Les faits mentaux sont donnés tels qu'ils sont dans l'éprouver (Erleben). Depuis la plénitude de l'expérience propre, l'expé- 
rience hors de nous est reproduite par transposition et ainsi comprise (GW, 264).

Une démonstration plus raffinée du processus de compréhension se trouve dans l'Aufbau où Dilthey constate que l'individualité déborde infiniment par ses possibilités ce qu'elle a réalisé et peut réaliser d'elle-même. Et c'est par la connaissance imaginative de ces possibilités qu'elle peut se représenter des individualités étrangères qui sont la réalisation effective de ces possibilités (cf. AGW, 259). C'est ici tout l'ordre du possible humain qui devient l'élément commun et le champ de référence transcendantal de la compréhension.

Dilthey était cependant trop bien averti des problèmes épistémologiques contemporains pour fonder une théorie des sciences humaines sur la seule thèse de l'immanence de son objet :

Le comprendre présuppose un éprouver (Erleben) et l'Erlebnis ne devient expérience de vie qu'en autant que le comprendre conduise au-delà de l'étroitesse et de la subjectivité de l'éprouver dans la région du tout et du général (AGW, 143).

L'objet des sciences humaines, selon Dilthey, ce sont les opérations et les auvres de l'esprit humain, qui trouvent leur expression individuelle dans l'art, la religion et la philosophie $^{4}$, et leur expression collective dans les institutions socio-politiques : « faits de mœurs et de droit, de la science et de l'État, de la vie historique et sociale » $(\mathrm{GW}, 49$, cf. AGW, 145). Or, la connaissance ou compréhension de ces diverses manifestations de l'esprit ne peut se faire par la seule étude de leur contenu objectal suivant une méthode structuraliste, mais par un retour aux opérations originelles dont elles proviennent: c'est la méthode herméneutique. Dilthey est explicite à ce sujet :

« Les sciences de la nature sub-ordonnent à leurs moyens de construction en produisant par abstraction la similarité des phénomènes à ordonner avec ces moyens de construction.

4. "Même ce que Hegel distinguait en tant qu'Esprit absolu de l'esprit objectif : art, religion et philosophie tombe maintenant sous ce dernier concept, et c'est précisément en eux que l'individu créateur se montre en même temps comme représentant de ce qui est commun (von Gemeinsamkeit), et c'est justement dans leurs formes puissantes que l'esprit s'objective et il est connu par elles" (AGW, 151 ). 
Les sciences de l'Esprit, au contraire, ordonnent avant tout et principalement en ce qu'elles retranscrivent la réalité historique et sociale qui s'étend sans mesure, telle qu'elle nous est donnée seulement dans son apparaître extérieur ou dans ses effets ou comme simple produit, comme dépôt de vie objectivée, dans la 'vitalité' spirituelle dont elle est issue » (GW, 265) .

Le rapport entre ce que Dilthey appelle le «monde de l'esprit " ou «esprit objectif » et l'interliaison vitale dont nous aurons à parler plus loin se trouve ainsi posé d'avance. C'est dans cette Lebendigkeit essentielle ou interliaison vitale que se trouvent les lois qui gouvernent ses productions. Mais retourner à la vitalité spirituelle, c'est revenir à l'immédiateté des Erlebnisse, et le cercle de l'immanence risque de se fermer sur lui-même. Comment, sans sortir de l'immanence de la pensée, dégager des lois de la pensée qui permettent d'expliquer scientifiquement toute la diversité des formes de vie humaine dans l'histoire, et cela de telle manière que ces formes culturelles elles-mêmes s'y reconnaissent?

Il faut nous mettre avec Dilthey à la recherche des possibilités de généralisation incluses dans l'immanence de la vie de pensée, faute de quoi la réalité humaine ne pourra être connue que comme un phénomène extérieur, c'est-à-dire comme un phénomène non humain, ce qui fausserait dès le départ sa nature essentielle. Si la démonstration de ces possibilités de généralisation était faite, l'immédiateté de l'expérience interne vécue pourrait sans doute se passer d'une justification propre, puisque l'immanence qui traverse le processus entier aurait atteint son but: l'intelligence des phénomènes humains collectifs dont le tissu complexe constitue l'histoire. C'est à établir cette démonstration que s'attache une bonne partie de l'œuvre de Dilthey: "Les sciences [systématiques de l'esprit] tout autant que l'histoire dépendent pour leur assurance de ce que la compréhension du singulier puisse être élevée au niveau d'une validité universelle ${ }^{6}$."

5. Le terme "vitalité " (Lebendigkeit) doit être entendu ici au sens substantiel de vie, et non en un sens qualitatif. Cf. autre formulation très voisine dans AGW, 119-120.

6. «La genèse de l'herméneutique ", in GW, p. 317. 
Élever à un niveau de validité universelle, c'est nécessairement subsumer sous des lois. Or, ce qui tient lieu de lois de la vie psychique, ce sont les ensembles reliés en lesquels les Erlebnisse d'eux-mêmes s'organisent. On pourrait mettre en doute l'existence même de ces ensembles reliés ou interliaisons psychiques (psychische Strukturzusammenhänge), mais Dilthey prétend qu'ils offrent prise à la constatation empirique grâce aux fins concrètes qu'ils poursuivent et réalisent et qui constituent par anticipation le principe de leur unité et de leur signifiance. Les sciences de l'esprit sont donc des sciences par les fins, et non des sciences par les causes. Dans la mesure où l'histoire humaine peut être comprise, elle ne pourra l'être qu'à travers les buts que les collectivités elles-mêmes ou dans la pensée de leur dirigeants se seront fixés. Qu'est-ce que l'histoire du Québec, par exemple, si ce n'est celle des buts atteints et des buts manqués que la communauté française a poursuivis dans une colonie vaincue et intégrée politiquement et économiquement à l'Empire britannique?

La compréhension historique se situe consciemment pour Dilthey dans une autre perspective que celle d'une déduction des enchaînements de causes en histoire. Elle vise plutôt une sorte de causalité à rebours qui semble être la seule possible en ce domaine (cf. GW, p. 38) ${ }^{7}$.

Or les buts renvoient d'abord à des démarches de l'esprit, et non à des conditions matérielles qui n'ont qu'indirectement valeur de cause. Nous sommes ainsi ramenés, pour une compréhension scientifique de l'histoire, non pas à des lois causales, mais à la structure psychique comme fondement de toute généralisation.

Comment s'opère ce mouvement de retour vers des interliaisons psychiques étrangères qui caractérise toutes les reconstitutions historiques? Le point de départ en sera l'esprit objectif $^{8}$. Pour Dilthey, l'esprit objectif comprend tout ce que

7. "Il n'y a dans le monde historique aucune causalité d'ordre naturel et scientifique, car la causalité comprise en ce sens implique qu'elle produit des effets avec nécessité selon des lois ; l'histoire ne connaît que des rapports d'agir et de subir, d'action et de réaction" (AGW, 197).

8. "Le sepricisme historique ne sera surmonté que si la compréhension de systèmes spirituels (geistiger Gebilde) prend la place des raffinements psychologiques. Les premiers se trouvent là dans un objectivé extérieur et peuvent faire l'objet d'un comprendre systématique (kunstmässig) » (AGW, 260). 
Hegel renferme dans ce concept, plus les manifestations de l'Esprit absolu que sont l'art, la religion et la philosophie. Ce ne sont plus des expressions de la Raison universelle, mais de la « vie dans sa totalité ». Le passage de la vie à l'histoire se fait à travers une série d'interliaisons (Zusammenhänge) qui s'emboîtent les unes dans les autres depuis la vie psychique élémentaire jusqu'aux formes complexes de la vie historique. L'interliaison caractérise d'abord la vie même en tant qu'elle s'organise en totalités signifiantes centrées sur un but. On a donc à l'origine l'interliaison vitale (Lebenszusammenhang) qui prend chez l'homme la forme d'interliaisons psychiques des expériences internes (psychische Strukturzusammenhänge). Groupés en sociétés, les hommes tissent entre eux un réseau de relations auquel Dilthey donne le nom de «monde spirituel» (die geistige Welt), défini comme " complexe d'interaction (Wirkungszusammenbang) ou interliaison contenue dans ses produits permanents ». On peut supposer que les produits permanents du complexe d'interaction constituent l'esprit objectif dont il a été question plus haut. Les mondes spirituels s'intègrent à leur tour dans des ensembles socio-historiques plus vastes, aussi bien sur le plan diachronique que sur le plan synchronique, tels la nation, la période, l'époque, et, à ce niveau encore, ce sont les motivations et les fins communes qui servent de principe d'unification en ensembles reliés signifiants ou interliaisons de signification (Bedeutungszusammenbänge). C'est la tâche de l'historien de dégager ces multiples interliaisons de manière à reproduire l'interliaison historique ellemême (der bistorische Zusammenhang) comme totalité significative9. Son objet empirique (l'interrogé, das Befragte, de Heidegger) est l'esprit objectif saisissable dans ce que Dilthey appelle « les interliaisons structurelles des unités de vie » qui permettent de faire le pont entre la singularité des Erlebnisse, d'une part, et l'universalité de l'histoire, d'autre part, universalité entendue non en un sens formel, mais en ce sens que l'histoire reproduit l'esprit humain (cf. AGW, 150-151) ${ }^{10}$. Il

9. «L'histoire n'est que la vie, appréhendée du point de vue du tout de l'humanité et formant une interliaison " (AGW, 256).

10. "Dans le comprendre d'un produit historique comme l'expression d'un intérieur, il n'y a pas déjà une identité logique, mais le rapport propre d'une égoïté contenu dans différents individus » (AGW, 259). 
apparaît important d'introduire ici une citation plus longue de Dilthey résumant toute cette philosophie de l'histoire qui est en même temps une théorie de la connaissance historique. L'aboutissement de cette théorie consiste, dit-il,

en une conception du monde spirituel comme complexe d'interaction (Wirkungszusammenbang) ou interliaison qui est contenue dans ses produits permanents. Les sciences de l'esprit ont leur objet dans ce complexe d'interaction et ses créations...

Ce complexe d'interaction se distingue de l'interliaison causale de la nature en ce que, selon la structure de la vie psychique, elle engendre des valeurs et réalise des buts . . . J'appelle cela le caractère téléologique immanent des complexes d'interaction de l'esprit. Par ce caractère, j'entends une interliaison de performances (Leistungen) qui a son fondement dans la structure d'un complexe d'interaction.

La vie historique crée. Elle est constamment active dans la génération de biens et de valeurs, et tous les concepts de ceux-ci ne sont que des réflexions de son activité ( $A G W, 153$ ).

C'est la tâche de l'analyse historique de découvrir dans les buts concrets, les valeurs, les modes de penser l'élément commun de conformité qui régit une époque. C'est justement cet élément commun qui détermine ensuite aussi les oppositions qui y règnent.

Ainsi toute action, toute pensée, toute production en commun, bref chaque partie de ce tout historique a sa signifiance (Bedeutsamkeit) dans son rapport au tout de l'époque (AGW, 155).

On se rend compte que les lois hypothétiques sous lesquelles les phénomènes physiques sont subsumés dans les sciences de la nature sont remplacées dans les sciences de l'esprit par des ensembles structuraux appelés Zusammenhänge dont la signifiance est assurée par les fins poursuivies et à l'intérieur desquels les événements singuliers peuvent se comprendre, sinon s'expliquer, par leurs relations à ces totalités.

Il est frappant que Dilthey ne distingue pas les deux types de sciences par la structure logique de leur raisonnement (modèle Popper / Hempel), mais par la façon d'atteindre ou de constituer leur objet. 
Les deux classes de sciences s'accordent au fond entre elles seulement sur ce qui peut résulter, à partir de règles générales, de la connaissance d'un ensemble de faits dans lequel l'individuation du réel se produit sur la base d'uniformités.

Toute élaboration semblable exige la subordination du particulier au général, du fait singulier dans un ensemble relié (Zusammenhang). Celui-ci est, dans les sciences de la nature, un système imputé (untergelegt), logiquement transparent, de moyens de construction. Dans les sciences de l'esprit, c'est l'interliaison psychique donnée dans l'expérience interne et qui se situe dans des conditions de vie...

L'idéal des sciences de l'esprit est la compréhension de toute l'individuation humaine-historique à partir de l'interliaison et de la communauté de toute vie psychique (GW, p. 265).

Dans les sciences de l'esprit comme dans les sciences de la nature, il s'agit d'une inscription du singulier dans le général. Toutefois les deux démarches se distinguent par trois aspects :

$1^{\circ}$ le singulier est, dans un cas, du vécu, dans l'autre, de l'observé ;

$2^{\circ}$ le général est, d'un côté, interliaison psychique immanente à l'objet, de l'autre côté, la loi universelle ;

$3^{\circ}$ le rapport du singulier à l'universel est, dans les sciences de l'esprit, la relation de la partie au tout interrelié ; dans les sciences de la nature, celle du cas particulier à une loi générale.

Ainsi les sciences de l'esprit ont-elles leur ultime fondement non pas dans les rapports sociaux, économiques ou politiques eux-mêmes, mais dans la dimension psychologique ou psychique de l'homme. C'est l'universalité des structures du penser et du sentir, ce que Dilthey nomme «l'identité de la raison » (GW, 250), qui fonde la possibilité de la vie sociale et, en définitive, de l'histoire elle-même :

Ces réalités psychiques nous sont compréhensibles jusqu'en leur dernier fondement. Elles sont toutes apparentées les unes aux autres.

Validité générale des processus de pensée, transférabilité des sentiments, interdépendance des activités visant un but rendent possible l'interliaison de ces processus internes en un monde social et historique ( $\mathrm{GW}, 249)$. 
C'est ce genre d'affirmations concernant les conditions psychiques a priori de la constitution et de la connaissance des phénomènes humains sociaux-historiques qui font penser à une relecture phénoménologique de la déduction transcendantale de la Critique de la raison pure. Bien qu'il démarque périodiquement sa démarche par rapport à celle de l'idéalisme transcendantal, il arrive assez souvent à Dilthey de trahir son hérédité :

Et partout dans ces sciences de l'esprit peut se démontrer la conjugaison de l'expérience externe, de l'expérience interne, de la méthode transcendantale et de la transposition de l'expérience interne dans les objets sur le fondement de la similitude de la vie de l'esprit ( $G W, 251)$.

Dans l'universalité du penser et du sentir s'inscrit également l'identité du vouloir avec la reconnaissance des valeurs et des buts, et nous nous retrouvons ainsi, au niveau des sciences humaines, en face d'une «fondation de la métaphysique des mœurs ", associée aux conditions de possibilité de la connaissance elle-même. Une fois présent à l'esprit l'ensemble de cette énorme construction, on devra, bien entendu, se poser la question de son fondement et de sa valeur scientifique.

\section{À propos de certaines critiques}

Pour opérer cette vérification, nous avons choisi de passer par la médiation des commentaires critiques de H.-G. Gadamer et de Jurgen Habermas.

Le premier s'en prend à Dilthey aux pages 205 à 228 de l'édition allemande de Vérité et Méthode ${ }^{11}$. Je trouve assez inconfortable de me mesurer à ce cyclope tranquille qu'est le professeur Gadamer. Aussi cette brève discussion voudra-t-elle plutôt soulever quelques questions que s'ériger en confrontation.

Gadamer voit chez Dilthey une « discrépance » irréductible entre ses préoccupations épistémologiques - axées sur une conception cartésienne de la certitude scientifique - et son point de départ dans une philosophie de la vie (WM 224).

11. Tübingen, 1960. 
Il lui reproche également d'être à la fois à proximité et à distance de l'hégélianisme: trop proche parce que la conscience historique, chez Dilthey, ne serait qu'une forme historicisée de l'Esprit absolu, en laquelle Gadamer retrouve la même infinitude ou la même aptitude à franchir toutes les limites qui lui sont imposées (cf. WM 219); trop loin parce que cette conscience historique, qui n'est qu'une émergence de la vie elle-même, échappe à la systématisation conceptuelle qui est le sens propre de la philosophie: "Ce n'est pas dans le savoir spéculatif du concept, mais dans la conscience historique que s'accomplit le savoir de soi de l'Esprit "(WM, 216).

La conception que se fait Dilthey de l'histoire est-elle vraiment trop hégélienne pour servir de base à une épistémologie de la connaissance historique ? À propos de la formule diltheyienne: "le travail formateur de pensée de la vie" (" die gedankenbildende Arbeit des Lebens », AGW, 136), Gadamer commente :

Ce qui distingue cette expression de Hegel n'est pas aisé à dire ... En autant que la vie est comprise en fonction de la pensée qu'elle forme, elle est soumise à un schème d'interprétation téléologique et est pensée comme esprit. Avec cela s'accorde le fait que Dilthey, dans ses dernières années, s'appuie de plus en plus sur Hegel et parle d'esprit là où auparavant il parlait de vie (WM, 214).

Disons tout d'abord que cette critique perd quelque peu de son poids du fait que la vie, pour Dilthey, était déjà au départ la vie réfléchie: « J'emploie, dit-il, le terme vie dans les sciences de l'esprit en le restreignant au monde humain ${ }^{12}$. »

Mais ce qui paraît décisif, c'est que Dilthey oppose explicitement à Hegel l'idée à tonalité marxisante que l'esprit est un produit social :

L'erreur de Hegel, c'est qu'il construit dans l'immanence les étapes de l'Esprit, alors qu'elles procèdent de l'action combinée de ce moment (i.e. l'intériorisation) avec la situation historique. Il est dans son intériorité de pensée même le produit du mouvement des lois du monde social (AGW, 271) ${ }^{13}$.

12. "Ich gebraucbe den Ausdruck Leben in den Geisteswissenschaften in der Einscbränkung auf die Menschenuelt $"(\mathrm{AGW}, 228)$.

13. Ou encore « À rejeter également les extrapolations qui se présentent là où l'on construit des hypothèses positives sur une relation de la conscience propre à une unité réelle qui la 
Il est aisé de constater que Dilthey se distingue radicalement de Hegel en ce qu'il considère les idées ou idéaux comme termes d'une évolution socio-historique, et non comme son principe, si bien que les «figures de l'esprit» ne peuvent aucunement guérir de leur relativité par un rapport à l'Absolu (cf. AGW, 289-291). S'il y a encore là du Hegel - ce qu'il paraît difficile de contester -, c'est un Hegel qui a subi une importante chirurgie plastique peut-être sous les influences secrètes représentées par Marx et Nietzsche. Dilthey n'hésite pas à affirmer que « la factualité irrationnelle est la base de l'histoire » (AGW, 288).

En outre, Dilthey ramène tout le contenu de l'Esprit absolu de Hegel au niveau concret de l'esprit objectif, ce dont Gadamer est certes conscient (WM, 215), mais qui semble moins le rassurer que l'inquiéter, puisque l'objectivité de l'esprit se perd, selon lui, dans la conscience historique. Sans doute la conscience historique "s'élargit-elle dans l'universel ", mais seulement "dans la mesure où elle comprend toutes les données de l'histoire comme expression de la vie dont elles procèdent ". "La vie saisit ici la vie », selon le mot de Dilthey lui-même. Comment la conscience historique se mirant elle-même dans son ouvre peut-elle fonder une science de l'histoire selon un modèle de clarté cartésienne (WM, 216)?

dépasse, que l'on détermine cette unité en termes de psychologie des peuples ou de transcendance " (AGW, 285).

Dans le paragraphe qui suit immédiatement cette citation, Dilthey va jusqu'à considérer la spéculation de l'Idéalisme transcendantal comme consacrant la mort de l'histoire :

"La conclusion qui passe du fait de la conscience de l'appartenance commune et de la validité générale dans la pensée et le vouloir à un sujet méta-empirique qui se manifeste dans la conscience individuelle, caractérise le point tournant de la spéculation kantienne vers la méthode constructive transcendantale. Celle-ci place sous les faits d'interliaison, d'appartenance commune, d'échangeabilité de la position des concepts, du lien basé sur l'obligation, une interliaison réelle qui doit rendre ces relations explicables.

La création de cette métbode transcendantale est la mort de l'bistoire puisqu'elle exclut justement la possibilité de s'enfouir dans les réalités mentionnées par le moyen de concepts historiques féconds. Également à rejeter la transgression qui, en quelque sens que ce soit, met des sujets réels sous les appartenances de type historique tu lieu den faire des porteurs d'énoncés à titre de sujets logiques qui soient différents de ceux de la psychologie individuelle " ( $A G W, 285$ : les italiques sont de nous).

"Il faut reconnaître que la toile de fond de ces idées (i.e. propres à chaque époque) est la violence qui ne peut être surmontée par ce monde supérieur . . . Factualité de la race, de l'espace, des rapports de violence constitue partout le fondement non spiritualisable. C'est un rêve de Hegel de croire que l'époque représente une étape du développement de la raison. Il y a cependant une interliaison interne qui conduit des relations conditionnantes, des factualités, de la lutte des forces jusqu'au développement des idéaux » (AGW, 287-288). 
Nous avons essayé de montrer qu'il y a pour Dilthey d'autres constructions que celles des systèmes conceptuels de type positiviste, voire même que ceux-ci dérivent de structures plus originaires ${ }^{14}$. N'est-ce pas justement l'objet de la recherche de Dilthey qu'une édification du monde historique selon un mode de connaissance qui lui serait propre et qui légitimerait autrement les sciences de l'esprit? S'il est vrai, comme le pense Gadamer, que Dilthey cherche à travers la vie consciente le même type de certitude (type cartésien) que dans les sciences dites exactes (WM 225-226), cette critique pourrait être décisive. Mais l'effet produit par l'ensemble de l'œuvre semble indiquer, au contraire, que Dilthey est surtout préoccupé par la différence plutôt que par la similitude, la certitude dite cartésienne ne lui servant que de point de repère.

Il faut noter encore cette différence essentielle qu'il n'y a pas pour Dilthey d'Esprit ou d'Idée en-soi en quête de son autre achevé, de sa figure définitive qui serait cette totale transparence de soi à soi qu'est le savoir absolu. De cela Gadamer est également conscient. Mais même l'esprit objectif a un autre statut et une autre signification. Il semble ne constituer pour Dilthey que la vérification du fait que la vie est une puissance structurante, c'est-à-dire qu'elle engendre des interliaisons dans lesquelles les singularités s'organisent autour d'un axe téléologique et qu'elle est par conséquent susceptible d'une interprétation (herméneutique) rigoureuse, puisque ces interliaisons trahissent sa normativité intrinsèque. S'il y a là un référent idéaliste, il me paraît être davantage de type kantien que de type hégélien, mais il n'est pas indispensable qu'il soit l'un à l'exclusion de l'autre. C'est donc ainsi que nous répondrions à la question posée par Gadamer : "Si la vie est cette réalité inépuisable et créatrice, telle que Dilthey se la représente, est-ce que le changement constant de l'interliaison de signification de l'histoire n'exclut pas un savoir qui atteigne l'objectivité ? (WM 217). Nous pensons que non. Le savoir qui en résulte, toutefois, n'est ni le savoir spéculatif de Hegel, ni les modèles opératoires de la science, mais la compréhension

14. C'est, du reste, ce qui permettra à Heidegger de considérer son analyse existentiale comme un prolongement des intentions de Dilthey (Sein und Zeit, Max Niemeyer Verlag, Tübingen, 1967, p. 377, 397-398. 
herméneutique de totalités, centrées sur un but. La critique de Gadamer semble osciller entre deux reproches dont il n'est pas sûr qu'ils soient conciliables. Dans la citation ci-dessus, il se demande si la conscience historique est susceptible d'un savoir qui atteigne l'objectivité. Plus loin, c'est la préoccupation inverse qui se fait jour. L'herméneutique romantique aurait permis à Dilthey " de mettre en accord le mode de connaissance des sciences de l'esprit avec les critères méthodologiques des sciences de la nature ", mais "cela n'a pu lui réussir sans ignorer l'historialité propre et essentielle des sciences de l'esprit "(WM 226-227). On ne voit plus très bien si, d'après Gadamer, Dilthey réussit ou ne réussit pas à contraindre son objet à l'intérieur des normes de la science, autrement dit, s'il sacrifie ces dernières ou s'il immole son objet.

Habermas s'éloigne, dès le départ, de la critique de Gadamer du seul fait qu'il reconnaisse chez Dilthey la volonté de définir le statut épistémologique propre des sciences de l'esprit :

Dilthey prend sur lui la tentative de démontrer une telle position méthodologique particulière des sciences de l'esprit (El 179).

Ce statut épistémologique particulier ne tient pas à la présence d'une activité subjective qui serait absente du comportement scientifique, puisque ce dernier suppose aussi une ingérence du sujet dans la mesure même où il soumet les phénomènes naturels aux conditions de la mesure selon les catégories du nombre, de l'espace, du temps et de la masse. Ce n'est pas tant l'objectivité qui se trouve réduite dans les sciences de l'esprit que l'objectivation conçue comme subordination de l'objet. Selon l'expression de Dilthey lui-même, ces dernières sciences ordonnent là où les premières subordonnent. Dans les sciences de l'esprit, les théories ne visent qu'à « la production d'un Erlebnis qui ré-effectue » l'expérience passée (Hervorbringung eines nachvolliziehenden Erlebnisses, p. 183). C'est toute la différence entre expliquer et comprendre. Dans un cas les interliaisons sont construites sur la base de modèles hypothétiques, dans l'autre elles sont données comme telles et revécues. Comprendre, c'est alors « transposer son propre soi 
dans un extérieur (ein Äusseres) de telle manière qu'un Erlebnis passé ou étranger redevienne présent dans le sien propre" (186). Habermas montre bien, toutefois, en s'appuyant sur une citation de Dilthey, que l'expérience revécue n'est pas immédiate, mais passe par la médiation obligée d'interliaisons objectivées dans les institutions, les mœurs, les œuvres d'art, etc. Dans les mots de Dilthey c'est "la régression vers un système spirituel (auf ein geistiges Gebilde) à partir d'une structure et d'une normativité (Gesetzmässigkeit) qui lui est propre " (AGW 85). Néanmoins, remarque Habermas, les interliaisons objectives ne livrent leur signification qu'à travers une reconstruction vécue dans laquelle nous rentrons dans le processus même de la production de sens (187). Nous avons nous-même relevé cette circularité menaçante dans notre analyse des textes de Dilthey. Or, dans la mesure où cette rentrée dans le processus de production de sens se fonde sur le principe de Vico qu'ici le sujet de connaissance prend lui-même part à la production des objets de sa connaissance, Habermas y décèle un autre cercle vicieux.

Les étapes logiques qu'il y distingue sont les suivantes :

1) Le principe de Vico selon lequel, dans les sciences humaines, «le sujet connaissant prend part à la fois à la production de l'objet de sa connaissance ».

2) «Les jugements synthétiques universellement valables de l'bistoire » rendus possibles sur la base du principe de Vico.

3) Le modèle diltheyien, établi par « les jugements synthétiques a priori sur ce qu'est l'histoire», "modèle d'un esprit qui s'objective et réfléchit en même temps ses expressions vitales $»$.

4) Conclusion: le cercle se ferme en pétition de principe, puisque le modèle diltheyien sous-tend déjà le principe de Vico.

On retrouve ici sous une autre forme la conception monadologique de l'herméneutique déjà soulignée par l'auteur. Cette argumentation n'est toutefois rendue possible que grâce à une "oversimplification" de la position de Dilthey que 
Habermas corrigera, du reste, un peu plus loin. Nous répliquerions donc comme suit :

1) Le modèle diltheyien ne fonde pas ni ne veut fonder le principe de Vico : ce n'en est qu'une formulation idéalisante où le sujet connaissant est remplacé par une conscience historique plus universelle, l'esprit.

2) Dans le modèle diltheyien, le sujet connaissant constitue un troisième terme face à l'esprit et à l'histoire ${ }^{15}$.

3) Les structures «transcendantales » s'objectivent dans l'histoire sous forme d'interliaisons qui découvrent l'universalité de l'esprit humain.

4) L'historien ou sujet connaissant a alors devant lui l'objet de son étude et les structures nécessaires à sa constitution comme objet de science ${ }^{16}$.

Ce qui semble toutefois rassurer Habermas quant aux limites de cette perspective, c'est que, pour Dilthey, la subjectivité individuelle ne se développe elle-même comme histoire et unité de signification, comme ensemble interrelié qu'en intercommunication avec les autres par le langage qui est le «medium dans lequel des significations sont partagées non seulement en un sens cognitif, mais dans le sens englobant d'une signifiance embrassant également des aspects affectifs et normatifs » (El 198). C'est par là, du reste, comme Habermas le signale en note (ibid., note 142) que «Dilthey fonde le primat de l'herméneutique au sens d'une interprétation systématique (kunstmässig) de l'expression langagière ». Bien entendu, Habermas se sent ici à domicile et sa critique de Dilthey, d'un point de vue épistémologique, s'en trouvera moins radicale que celle de Gadamer :

La "communauté des unités de vie ", que Dilthey conçoit comme étant l'esprit objectif, est caractérisée par une double dialectique du tout et de ses parties : sur le plan horizontal de

15. «Et ce n'est pas à la fois le même sujet qui éprouve et qui appréhende, mais l'individu se tient vis-à-vis le peuple, même lorsqu'il lui appartient, comme spectateur. Par là le comprendre devient un autre en vertu des catégories qui lui sont propres " (AGW, 282).

16. "L'historien forme à parrir du contenu des sources, de l'événement, le cours (de l'histoire), le complexe d'interaction "(AGW, 241). 
la communication par le rapport de totalité d'une communauté linguistique aux individus ... ; et verticalement dans la dimension du temps par le rapport de totalité d'une histoire de vie aux expériences vécues singulières et aux rapports vitaux à partir desquels elle se construit (El 200).

La rencontre dans une même structure dynamique de la diachronie et de la synchronie pourrait fournir la clef permettant d'échapper au cercle vicieux relevé plus haut, puisque le processus de formation de l'histoire de la vie individuelle se fait dans et par le langage qui fournit à tout Erlebnis son cadre quasi transcendantal. Aussi Habermas reconnaît-il que la communauté des unités de vie chez Dilthey sert de cadre objectif aux sciences de l'esprit tout comme chez Peirce, pour les sciences de la nature, l'ensemble du processus de recherche porté par la communauté des chercheurs. La compréhension des significations singulières doit passer par la médiation de catégories générales véhiculées par une communauté historique et linguistique structurée. Habermas croit trouver ici la condition quasi transcendantale de possibilité de chacune des interliaisons énumérées dans notre exposé sur Dilthey et de l'interconnexion de ces interliaisons entre elles, c'est-à-dire la communauté du langage. Et il cite à ce propos une affirmation de Dilthey :

Depuis l'interprétation élémentaire qui ne requiert que la connaissance de la significarion des mots et dè la réglementation selon laquelle ils sont reliés en phrases pour former un sens, et partant la communauté de langue et de pensée, le cercle de ce qui est commun et qui rend possible le processus de compréhension va en s'élargissant constamment (AGW 141).

Habermas me paraît, cependant, attribuer une place beaucoup trop centrale à la communauté linguistique dans la théorie de Dilthey. Certes, dans une perspective herméneutique, le langage comme expression est le pendant nécessaire de la compréhension, et comme expression commune de la compréhension des communautés et des individus. Mais $c e$ dont le langage est expression et qui est plus fondamental que lui, ce sont les interliaisons psychiques qui trahissent le mécanisme universel d'autostructuration de la vie dont l'autostructuration 
signifiante de l'histoire n'est qu'un mode au registre de la collectivité humaine ${ }^{17}$.

Les citations possibles à l'appui de l'interprétation que je défends ici sont légion. J'en saisis une au vol, qui pourrait bien être remplacée ou complétée par une multitude d'autres :

La signifiance est la déterminité de la signification (Bedeutung) d'une partie pour un tout et qui se produit sur le fondement du complexe d'interaction (Wirkungszusammembang) (AGW, 238).

La signifiance qui accueille ainsi le fait comme la déterminité du membre de signification à partir du tout est un rapport de vie, et non une relation intellectuelle, non une insertion de raison, de pensée dans une part de l'événement. İa signifiance est dégagée de la vie elle-même ( $A G W, 240)$.

Peut-on exprimer plus clairement que le lieu de la signification ne se situe pas dans le langage commun, mais dans les interliaisons structurelles qui seules rendent possible le langage lui-même ${ }^{18}$.

Ce qu'il y a encore de valable dans cette entreprise de Dilthey c'est, croyons-nous, son effort pour donner une forme systématique aux productions imprévisibles de l'esprit humain et les rendre ainsi accessibles à un mode de connaissance qui, pour n'avoir pas le caractère formel des théories scientifiques, n'en est pas moins universel, et cela, sans tomber dans le réductionnisme plus confortable peut-être, mais moins compréhensif et moins satisfaisant, des doctrines positivistes.

\section{Département de philosophie} Université de Sherbrooke

17. Toute la section $\$ 8$ de Connaissance et intérêt ne me parât se comprendre que par une certaine pression exercée sur les textes de Dilthey par la théorie habermasienne de l'intercommunication, bien que la seconde moitié de l'article risque de contredire la première en insistant sur l'absence de médiation dans l'herméneutique diltheiyienne.

18. "La signification ne dit rien d'autre que l'appartenance à un tout" (AGW, 230).

"Le même rapport (que dans une phrase) existe entre les parties et le tout d'un processus vital, et ici aussi la compréhension du tout, le sens de la vie vient de la signification ...

Par ce rapport, chaque Erlebnis singulier est recueilli de manière signifiante à partir d'un tout. Et tout comme les mots d'une phrase sont reliés à sa compréhension, ainsi l'incerliaison des Erlebnisse produit-elle la signification du cours de la vie. Il en va de même également pour l'histoire " ( $\mathrm{AGW}, 255)$. Cf. également le développement de la p. 278 où le langage apparaît comme un phénomène social à côté d'autres. 


\section{BIBLIOGRAPHIE}

DILTHEY, Wilhelm. Die Geistige Welt. Erste Hälfte. Abhandlungen zur Grundlegung der Geisteswissenschaften, Gesammelte Schriften, Band V. Vandenhoeck \& Ruprecht, 1974. Sigle: GW. Der Aufbau der Geschichtlichen Welt in den Geisteswissenschaften. G. unveränderte Auflage, Vandenhoeck \& Ruprecht in Göttingen, 1973. Sigle: AGW.

GADAMER, Hans-Georg. Wabrbeit und Metbode. Tübingen, 1960, p. 162-261. Sigle: WM.

HABERMAS, Jürgen. Erkenntnis und Interesse. Suhrkamp Verlag, Frankfurt a.m. 1978, 178-203. Trad. fr., Connaissance et intérêt. NRF, Gallimard, 1976 : Cf. Article intitulé « La théorie de la compréhension d'expression chez Dilthey », p. 175-195, de même que l'article suivant: "Autoréflexion des sciences morales : la critique historiste du sens ", p. 196-219. Sigle : EL.

MISCH, Georg. «Vorbericht des Herausgebers ", in Die Geistige Welt, p. VII-CXVII.

N.B. Toutes les traductions sont de nous. 